\title{
Reflexões e propostas para a estruturação de programas de Mestrado em Medicina de Família e Comunidade no Brasil
}

\author{
Reflections and proposals for the establishment of Family \\ and Community Medicine Master's Programs in Brazil
}

Leandro David Wenceslau (https://orcid.org/0000-0001-8622-9137) ${ }^{1}$

Thiago Dias Sarti (https://orcid.org/0000-0002-1545-6276) ${ }^{2}$

Thiago Gomes da Trindade (https://orcid.org/0000-0001-8178-0982) ${ }^{3}$

\footnotetext{
${ }^{1}$ Departamento de Medicina e Enfermagem, Universidade Federal de Viçosa. Av. P. H. Rolfs s/n, Campus Universitário. 36570-000 Viçosa MG Brasil. leandro.david@ufv.br ${ }^{2}$ Departamento de Medicina Social, Universidade Federal do Espírito Santo. Vitória ES Brasil.

${ }^{3}$ Departamento de Medicina Clínica, Universidade Federal do Rio Grande do Norte. Natal RN Brasil.
}

\begin{abstract}
While Primary Health Care (PHC) and Family and Community Medicine (FCM) have expanded their space and relevance in health care and undergraduate medical courses, Brazil has no stricto sensu FCM postgraduate programs. In this paper, we analyze some aspects of the Brazilian public health field and the national stricto sensu postgraduate system that can help to explain this scenario. As a contribution to the debate on this topic, we also gathered information from international postgraduate and research experiences in FCM and devised a curriculum proposal for future national FCM master's courses. In the end, we discussed some key strategies for the emergence of stricto sensu postgraduate courses in this discipline in Brazil, highlighting the potential of these programs for evaluation and qualification of primary care services, especially the Family Health Strategy, and the training of PHC specialists required for the consolidation of the Unified Health System (SUS) as an accessible, comprehensive and equitable health system for the Brazilian population.
\end{abstract}

Key words Family and community medicine, Postgraduate education, Research
Resumo Embora a atenção primária à saúde (APS) e a medicina de família e comunidade (MFC) tenham aumentado seu espaço e relevância no cenário assistencial e nos cursos de graduação médica, não há cursos de pós-graduação "stricto sensu" em MFC no Brasil. Neste artigo, analisamos alguns aspectos do campo da saúde pública brasileira e da própria estruturação da pós-graduação "stricto sensu" nacional que podem ajudar a explicar este panorama. Como uma contribuição para o debate neste tema, também reunimos informações de experiências internacionais em pós-graduação e pesquisa em MFC e elaboramos uma proposta de currículo para futuros cursos nacionais de mestrado em MFC. Ao final discutimos algumas estratégias fundamentais para o surgimento de cursos de pós-graduação "stricto sensu" nesta área no Brasil, destacando o potencial desses cursos para a avaliação e a qualificação dos serviços de atenção primária, em especial a Estratégia Saúde da Família, e para a formação de profissionais especializados em APS necessários para a consolidação do Sistema Único de Saúde como sistema acessível, abrangente e equitativo para a população brasileira.

Palavras-chave Medicina de família e comunidade, Educação de pós-graduação, Pesquisa 


\section{Introdução}

O Brasil há décadas considera a Atenção Primária à Saúde (APS) como a base ordenadora de seu sistema de saúde público - o Sistema Único de Saúde (SUS), sendo que, mais recentemente, esta tem sido também a estratégia adotada por parte do sistema de saúde suplementar ${ }^{1,2}$. Dados de julho de 2019 do Ministério da Saúde mostram que $74,2 \%$ e $63,8 \%$ das pessoas estão potencialmente cobertas por equipes de Atenção Básica à Saúde em geral e de Estratégia Saúde da Família (ESF), existindo hoje mais de 43 mil equipes de ESF$^{3}$. Estes investimentos resultaram, dentre outras coisas, na redução da mortalidade infantil ${ }^{4}$ e por doenças cardiovasculares ${ }^{5}$, na redução das internações por condições sensíveis à APS (há conflito de evidências na literatura $)^{4}$ e na redução de iniquidades em saúde numa perspectiva racial ${ }^{6}$. Consolidar a APS no país, portanto, significa investir em melhores níveis de saúde da população, em especial da porção mais vulnerável, garantindo direitos constitucionais ${ }^{1}$.

Os desafios para o fortalecimento da APS no Brasil são conhecidos: subfinanciamento da saúde; fragmentação da rede de atenção à saúde; problemas na estrutura das unidades básicas de saúde; alta rotatividade dos profissionais das equipes; etc. ${ }^{1}$. Frisa-se, no entanto, o grave problema da qualificação dos profissionais que atuam na APS, independente de sua categoria profissional, mas com destaque para os profissionais médicos ${ }^{1}$. Dados de 2017 indicam que há aproximadamente 5.500 médicos de família e comunidade (MFC) titulados no país 7 . Se todos atuassem na ESF, o que está bem distante da realidade, teríamos apenas $12,8 \%$ das equipes contando com um MFC titulado, provavelmente distribuídos de maneira iníqua pelo território nacional.

Por outro lado, o reconhecimento que se tem da APS, e em especial da MFC, enquanto áreas acadêmicas com corpo de conhecimentos específico é incipiente em todo o mundo ${ }^{1,8}$. Assim como faltam especialistas em MFC para atuarem nos serviços, são poucos os MFCs inseridos nas graduações e pós-graduações, são escassos os pesquisadores que estruturam suas carreiras em torno da APS e da MFC, rareiam os departamentos de MFC e APS nas universidades ${ }^{1,9-11}$. Os editais de financiamento das agências brasileiras de fomento à pesquisa, até aqui, não têm contemplado de forma específica a medicina de família e comunidade, o que leva uma inconsistência de investimento nesta área de conhecimento no país. Cria-se um círculo vicioso no qual pouco se valoriza a pesquisa em MFC, logo poucos pesquisadores - médicos à frente - se interessam, fragilizando a área num contexto universitário de recursos escassos e relações de poder complexas.

É com base nesse breve diagnóstico que buscamos discutir neste artigo a relevância da pós-graduação stricto sensu em MFC como um dos pilares da consolidação desta área no país enquanto núcleo de conhecimento cientificamente válido e robusto e socialmente legítimo e necessário. A APS é, em sua essência, uma área interdisciplinar, porém, abordaremos a importância de uma estrutura acadêmica voltada para o desenvolvimento dos conceitos, competências e evidências científicas que nortearão a prática clínica em equipe na APS. Neste caso, o fortalecimento da MFC enquanto área de conhecimento e estrutura de formação pós-graduada stricto sensu é um caminho incontornável. Apresentaremos também algumas propostas para a estruturação de programas de mestrado em MFC no Brasil a partir de nosso contexto e problemática, trazendo para o debate informações e experiências do cenário internacional sobre este tema.

\section{Medicina de família e comunidade e a pós-graduação stricto sensu no Brasil}

A MFC, no Brasil, tem uma história de mais de 40 anos enquanto movimento institucional de formação de especialistas médicos ${ }^{12}$. Entretanto, o impulso mais significativo para a formação médica em MFC vai ocorrer já nos anos 2000, a partir da conjunção de uma série de fatores que envolve políticas públicas indutoras e reestruturação do mercado de trabalho médico ${ }^{1}$. Neste período, são criados diversos movimentos de reorientação da formação médica em nível de graduação com maior ênfase na APS; surgem políticas robustas de provimento médico na APS, como o Provab e o Programa Mais Médicos, com políticas indutoras da formação do MFC acopladas; municípios de grande porte reformulam suas políticas de saúde locais e criam PRMs em MFC com número de vagas expressivo; e a saúde suplementar expande sua cobertura populacional e passa, ao menos em parte, a incorporar experiências de APS com centralidade do MFC. Tais medidas resultaram em um significativo crescimento da MFC no país: segundo maior número de vagas de RM dentre todas as especialidades médicas, com mais de $10 \%$ das vagas totais; sétimo maior número de médicos cursando um PRM em 2017 dentre todas as especialidades; e um aumento significativo de especialistas aptos a atuarem nos 
últimos anos ${ }^{13}$. Infelizmente, ainda são desafios uma das mais altas proporções de vagas de RM ociosas, a baixa procura da especialidade como primeira opção pelos médicos recém-formados e um ainda baixo número de especialistas em relação ao total de médicos titulados $(1,4 \%)^{13}$.

No universo acadêmico, a realidade é também de muitos desafios para a MFC ${ }^{1,10,11}$. Apesar de ser crescente o número de MFCs vinculados às graduações médicas, são praticamente inexistentes departamentos da área nas universidades, em particular nas públicas. A MFC não é reconhecida como área de conhecimento pela CAPES (Coordenação de Aperfeiçoamento de Pessoal de Nível Superior), o que dificulta a construção de uma estrutura de pós-graduação e pesquisa própria. E não há programas de pós-graduação stricto sensu em MFC no Brasil. O resultado é que os MFC fazem suas pós-graduações e se inserem enquanto docentes em departamentos de outras áreas, em particular nas áreas médicas e na saúde coletiva. Até 2018, 747 MFC haviam concluído o mestrado (554 em programa acadêmico e 193 em programa profissional) e 170 haviam concluído o doutorado - respectivamente, 12 e $2,7 \%$ do total de MFCs brasileiros 9 . A falta de investimentos e linhas de financiamento de pesquisas específicas em MFC torna a produção científica na área mais difícil e provavelmente de baixa expressão numérica. Os pesquisadores com formação em MFC constroem suas carreiras em linhas de pesquisa vinculadas a outras áreas e temas, como pesquisas em serviços de saúde - health services research - que, apesar de serem importantes para o SUS e fazerem eventualmente conexões com a MFC, não aprofundam em seus atributos, reduzindo o impacto do conhecimento produzido no fortalecimento da APS. O resultado final é a ainda tímida inserção da MFC enquanto núcleo de conhecimento científico em saúde nas ciências brasileiras, o que está longe de ser uma particularidade nossa, mas posiciona mal o país no comparativo com sistemas de saúde de países mais desenvolvidos ${ }^{1}$.

O sistema de pós-graduação brasileiro é tido como um dos mais robustos e bem sucedidos do mundo, apresentando um expressivo crescimento quantitativo e qualitativo do número de programas e produção acadêmica ao longo das décadas $^{14-16}$. Dados da CAPES de $2018^{17}$ mostram que o Brasil tem 4.291 programas de pós-graduação ativos, a maioria oferecendo cursos de mestrado e doutorado ( $\mathrm{n}=2.186 ; 51 \%)$ em instituições federais de ensino superior $(n=2.472 ; 57,6 \%)$. As áreas médicas de avaliação possuíam 249 (5,8\%) programas e a saúde coletiva 89 (2,1\%). Contudo, são inúmeros os desafios que tensionam a política nacional de pós-graduação, sendo importante aqui citar algumas mais relacionadas ao objeto em análise. A expansão dos cursos de pós-graduação se deu de forma concentrada nas regiões Sudeste e Sul, o que afeta o desenvolvimento social regional. Atrelado ao histórico subfinanciamento da pós-graduação e das políticas educacionais e sociais em geral, estas desigualdades regionais reforçam um processo iníquo no qual as universidades mais bem posicionadas dos grandes centros urbanos do país têm maiores condições de captar recursos, de atrair pesquisadores qualificados, de atender as necessidades locais de formação profissional e conhecimento técnico-científico e de conectarem-se a redes de pesquisas internacionais ${ }^{14,15,18,19}$.

É importante frisar a enorme dificuldade de avaliar a política e os programas de pós-graduação ${ }^{18,20}$. De uma forma geral, o modelo de avaliação dos programas de pós-graduação vigente no Brasil exige do docente um grau de dedicação à pesquisa que gere resultados em sua produção acadêmica que podem impedi-lo de exercer outras funções sociais, o que pode dificultar a atração de pesquisadores em áreas como a medicina, seja pela alta exigência de dedicação de carga horária, seja pelo impacto financeiro em termos de rendimentos pessoais. Possivelmente aqui reside um dos maiores desafios para a inserção das médicas e médicos de família no cenário universitário e de pós-graduação.

O Brasil não tem tradição em pós-graduação em APS e MFC, sendo que a produção acadêmica destes núcleos de conhecimentos encontra-se difundida em outras áreas de conhecimento, em maior grau na Saúde Coletiva ${ }^{9}$, que vem se dedicando à produção de conhecimento em avaliação das políticas direta ou indiretamente relacionadas à APS, em organização de processos de trabalho em serviços de APS e em políticas de formação para a APS, principalmente em nível de graduação ${ }^{1}$. A forte ligação histórica da Saúde Coletiva com a APS cria um espaço acadêmico relativamente acolhedor para as demandas que a MFC se coloca no plano científico, algo mais complexo de ocorrer nas áreas médicas.

Há uma história de programas voltados à APS em algumas instituições de ensino, mas que não se configuram como um investimento massivo na área. São exemplos o Programa de Mestrado Profissional em APS da Universidade Federal do Rio de Janeiro; os Programas de Mestrado em Saúde da Família das Universidades Federal do 
Ceará e Mato Grosso do Sul, Fundação Oswaldo Cruz e Estácio; e os programas de Saúde na Comunidade das Universidades de São Paulo e Federal do Piauí ${ }^{21}$. Por outro lado, destacam-se duas experiências de mestrado profissional em Saúde da Família feitas em rede, com entradas de grande número de profissionais: os programas da Rede Nordeste de Formação em Saúde da Família (RENASF), criada em 2012, e o Mestrado Profissional em Saúde da Família (ProfSaúde), criado em $2015^{22,23}$.

Ou seja, de movimentos pontuais de pós-graduação com interfaces mais ou menos diretas, temos um novo processo de massificação da pósgraduação em APS a partir de instituições públicas de ensino e pesquisa. É evidente a crescente importância da APS no cenário assistencial e sanitário brasileiro, inicialmente com a expansão de cobertura populacional de serviços de saúde, posteriormente com a ênfase em nível de graduação em saúde, e mais recentemente na produção acadêmica. É chegada a hora do país contar também com um sistema de pós-graduação em Medicina de Família e Comunidade que dialogue com este movimento e faça avançar a área em suas especificidades.

\section{Mestrado em medicina de família e comunidade: construção de uma imagem objetivo}

A partir desta breve contextualização sobre o cenário da MFC e da pós-graduação stricto sensu no Brasil, incluindo as experiências recentes em Saúde da Família e APS, reunimos alguns dados e sugestões que podem subsidiar a organização de programas nacionais de mestrado em MFC. Para desenvolver esta tarefa, utilizamos três estratégias: (1) mapear e analisar experiências internacionais de mestrado em MFC já em curso; (2) pesquisar recomendações de instituições internacionais representativas da especialidade; e (3) identificar as exigências e as recomendações do sistema educacional brasileiro para a criação de novos cursos de pós-graduação stricto sensu em medicina de família e comunidade. Começamos pelo levantamento e análise de experiências internacionais já em curso.

Em diversos países da Europa Ocidental, América do Norte e Oceania, os médicos e as médicas de família e comunidade constituem um número significativamente maior da população total de médicos desses países quando comparados ao Brasil. Os MFCs - com a designação equivalente em cada país - somam $28 \%$ dos médicos no Reino Unido e Espanha, 48\% no Canadá e $45 \%$ na Austrália e na Holanda ${ }^{24,25}$. Entretanto, mesmo nesses países, o número de programas de mestrado ou doutorado em MFC é pequeno. Inicialmente, foram analisados os portais virtuais da World Association of Family Doctors (WON$\mathrm{CA}$, associação que congrega as diversas sociedades de especialidade em MFC do mundo) e da World Federation of Medical Education (WFME, organismo mundial que reúne instituições de ensino médico), porém, não foram encontrados, em seus sítios virtuais, bancos ou plataformas internacionais com informações específicas sobre programas de pós-graduação stricto sensu. Portanto, para chegar a essas informações, realizamos em outubro de 2019 uma busca em portais governamentais de educação superior de países reconhecidos por sua forte tradição em MFC e APS: Austrália, Canadá, Espanha, Holanda e Reino Unido. De forma a ampliar o alcance do mapeamento, pesquisamos também na plataforma Google ${ }^{\varpi}$, refinando a busca para localizar apenas sítios que continham, em seus títulos, as expressões exatas: "master of family medicine"; "master of science in family medicine"; ou "maestría en medicina familiar". Nas buscas em plataformas governamentais, foram identificados dois programas de mestrado no Canadá e um no Reino Unido. Não foram encontrados programas situados na Austrália, Espanha ou Holanda. Através da pesquisa na plataforma Google ${ }^{\bowtie}$, foram encontrados cursos em Chipre, Malta e Peru, um programa cada. Foram excluídos dos resultados programas designados pelas expressões exatas de busca, que, porém, apresentavam organização curricular semelhante a especializações clínicas lato sensu, com uma ampla carga horária obrigatória em disciplinas com foco assistencial ou de gestão, muitas vezes dispensando qualquer tipo de manuscrito de conclusão. Estes programas não correspondem ao que internacionalmente é designado como master of science (MSc), equivalente ao mestrado acadêmico ou profissional em saúde no Brasil. Foram encontrados programas de mestrado com este formato na África do Sul (nove programas, presentes em todas as universidades que oferecem bacharelado em medicina e cirurgia neste país ${ }^{26}$ ), Austrália (1), Malásia (1), Nicarágua (2), Paraguai (2), Singapura (1) e Sudão (1).

É provável, entretanto, que o número de programas de mestrado em medicina de família no mundo seja maior. As estratégias de levantamento adotadas neste trabalho possuem limitações, como a exclusão de sítios de programas apresen- 
tados em outros idiomas, que não espanhol ou inglês, e a ausência de contato direto com instituições internacionais de ensino de pós-graduação stricto sensu em medicina. Por exemplo, em pesquisa realizada através de questionários enviados a representantes de 313 escolas médicas da região da Ásia Pacífico, com uma taxa de resposta de $31 \%$, Jenn et al. ${ }^{27}$ identificaram 17 programas de mestrado em MFC (com destaque para 4 programas na Indonésia, 4 no Japão, 3 na Coréia do Sul e 3 na Malásia), formatados basicamente para elaboração de dissertações e teses. Esses dados, que são parciais, considerando a taxa de resposta, apontam para a necessidade de um cadastro global de informações sobre programas de pós-graduação stricto sensu em MFC que dê visibilidade aos programas existentes, possibilite o intercâmbio de experiências e conhecimentos entre eles e apoiem o surgimento de novos programas. Novos levantamentos podem adotar metodologias mais abrangentes como surveys internacionais de alcance global e consultas a informantes-chave de destaque no cenário científico da medicina de família no mundo.

Nos seis programas mencionados acima (do Canadá, Chipre, Malta, Peru e Reino Unido), a duração variou entre 1 ano e meio a 5 anos, a depender se o estudante opte por regime de dedicação integral ou parcial, quando é aceita essa segunda possibilidade. Em todos, existe um conjunto de disciplinas obrigatórias e três programas exigem também créditos em disciplinas optativas. Três cursos exigem a elaboração de uma tese ou dissertação para obter o título de mestre, dois exigem a elaboração de um projeto de pesquisa em medicina de família e, na Western University, o título de mestre pode ser obtido mediante a apresentação de uma tese, um projeto de pesquisa ou um ensaio, além da conclusão das disciplinas. Vale ressaltar que quatro programas conferem titulação específica para aqueles que concluem as disciplinas, mas não apresentam tese ou projeto de pesquisa ao final. Os cursos de Malta, do Peru e um curso do Canadá são abertos para profissionais de saúde em geral, os demais exigem, como pré-requisito dos candidatos, a graduação em medicina.

Apesar dessas convergências mais estruturais, os temas abordados pelas disciplinas obrigatórias variaram significativamente entre os cursos. A única semelhança entre todos foi a obrigatoriedade de ao menos uma disciplina de metodologia de pesquisa. Três programas também possuíram alguma disciplina obrigatória em fundamentação teórica da medicina de família e três, uma disciplina voltada para comunicação clínica e cuidados centrados no paciente. Dois programas incorporaram disciplinas voltadas para temas comuns da prática clínica da MFC e um programa, da McGill University, se destacou por ser composto basicamente por disciplinas voltadas para diversos métodos de pesquisa. Os dados estão sistematizados no Quadro 1.

A heterogeneidade da amostra estudada de programas e a lacuna de informações mais precisas sobre programas de mestrado em MFC no mundo tornam o desafio de pensar sugestões curriculares para futuros programas de mestrado em MFC no Brasil ainda mais complexo. Em 2013, o WONCA Working Party on Education publicou o documento WONCA Global Standards for Postgraduate Family Medicine Education ${ }^{28}$, que, no entanto, é voltado para orientar a formação em competências clínicas dos médicos e médicas de família em programas de residência ou especialização médica. Uma referência que, em parte, supre esta falta de exemplos norteadores e documentos balizadores de programas de pós-graduação stricto sensu é a série de artigos Research agenda for general practice/family medicine and primary health care in Europe $e^{29-33}$. Nestes artigos, publicados por membros da European General Practice Research Network, os resultados de revisões de literatura realizadas sobre a produção científica em temas vinculados a cada uma das seis competências essenciais da MFC definidas pela WONCA: gestão em atenção primária, abordagem abrangente, cuidados centrados no paciente, abordagem holística, competências específicas para solução de problemas e orientação comunitária. A partir dessas revisões, foram recomendados tópicos, questões e métodos de investigação que compõem uma proposta de agenda de pesquisa para a medicina de família na Europa, mas que pode servir como ponto de partida para outros continentes e que estão sintetizados no Quadro 2. A identificação dessas demandas de pesquisa próprias da medicina de família é fundamental para o desenho de currículos de pósgraduação que ofereçam a formação adequada para a realização de estudos nessa disciplina.

Retornando a especificidades do contexto brasileiro, é atribuído à $\mathrm{CAPES}^{34,35}$, que por sua vez distribui a comissões de cada uma de suas 49 áreas de avaliação, o papel de discriminar os requisitos para a aprovação de novos cursos. Portanto, um primeiro passo é definir uma área de avaliação a que devem estar vinculados os futuros programas de MFC. Considerando sua natureza essencialmente clínica, sugerimos que 


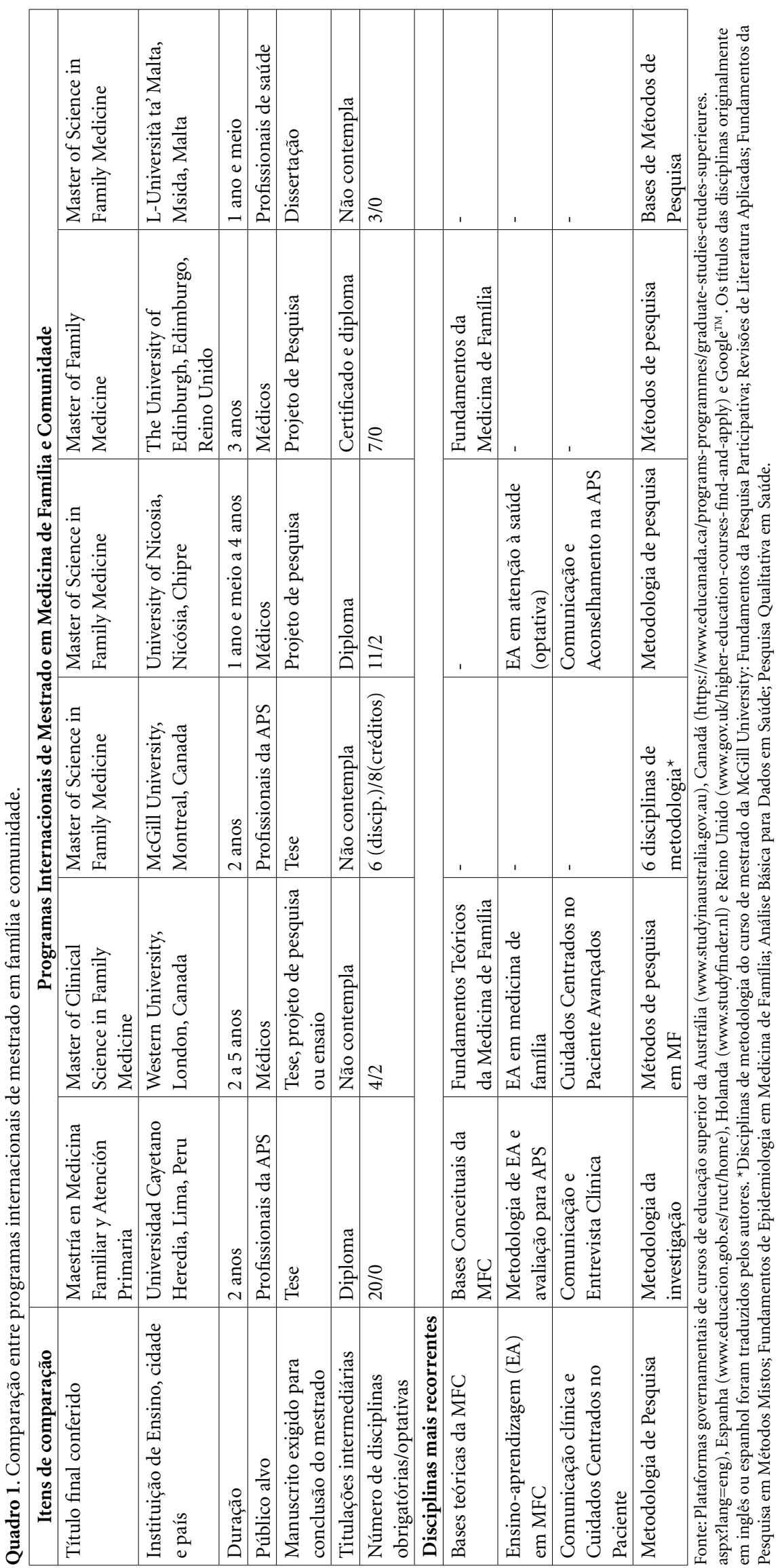


Quadro 2. Resumo adaptado das recomendações da agenda de pesquisa para a medicina de família da European General Practice Research Network.

\begin{tabular}{|c|c|c|}
\hline Categoria de pesquisa & Possíveis temas de pesquisa & Métodos de investigação \\
\hline $\begin{array}{l}\text { Gestão da atenção } \\
\text { primária à saúde }\end{array}$ & $\begin{array}{l}\text { - Comparação entre modelos de atenção primária à } \\
\text { saúde, incluindo modelos de organização da força } \\
\text { de trabalho e de financiamento } \\
\text { - Desenvolvimento e análise de indicadores de } \\
\text { custo-efetividade e qualidade } \\
\text { - Tempo de consulta, acessibilidade dos serviços, } \\
\text { cuidados colaborativos, encaminhamentos entre } \\
\text { médicos de família e outros especialistas } \\
\text { - O papel e o impacto dos prontuários eletrônicos } \\
\text { Formação em gestão na APS para médicos }\end{array}$ & $\begin{array}{l}\text { - Pesquisas para } \\
\text { desenvolvimento de } \\
\text { instrumentos para avaliação da } \\
\text { atenção primária } \\
\text { - Estudos epidemiológicos } \\
\text { longitudinais em medicina de } \\
\text { família. } \\
\text { - Estudos comparativos de } \\
\text { intervenções com diferentes } \\
\text { modelos de atenção primária. } \\
\text { - Pesquisas com métodos mistos. }\end{array}$ \\
\hline $\begin{array}{l}\text { Cuidados centrados } \\
\text { no paciente, } \\
\text { abordagem } \\
\text { abrangente e } \\
\text { abordagem holística }\end{array}$ & $\begin{array}{l}\text { - Aprimoramento dos conceitos e competências } \\
\text { que definem centralidade na pessoa, abrangência } \\
\text { (integralidade) e abordagem holística } \\
\text { - Desenvolvimento de indicadores e instrumentos } \\
\text { de avaliação dessas competências e de seus } \\
\text { componentes } \\
\text { - Compreensão de circunstâncias sociais, culturais e } \\
\text { ambientais que podem afetar a saúde, considerando } \\
\text { estes aspectos } \\
\text { - Percepções, perspectivas e preferências de } \\
\text { pacientes e médicos sobre comunicação, } \\
\text { centralidade da pessoa, relacionamento e tomada de } \\
\text { decisão compartilhada } \\
\text { - Avaliação de abordagens centradas na pessoa, } \\
\text { modelos abrangentes de cuidado, abordagens } \\
\text { holísticas utilizando desfechos clínicos e também } \\
\text { satisfação, compreensão dos pacientes e qualidade } \\
\text { de vida }\end{array}$ & $\begin{array}{l}\text { - Pesquisas qualitativas } \\
\text { - Pesquisas para o } \\
\text { desenvolvimento de indicadores } \\
\text { e instrumentos de mensuração } \\
\text { dessas competências } \\
\text { - Estudos longitudinais } \\
\text { prospectivos e retrospectivos } \\
\text { - Estudos de intervenções } \\
\text { voltadas para cuidados com } \\
\text { essas características } \\
\text { - Pesquisas com métodos mistos }\end{array}$ \\
\hline $\begin{array}{l}\text { Competências } \\
\text { específicas para } \\
\text { solução de problemas }\end{array}$ & $\begin{array}{l}\text { - Apresentação e evolução clínica de doenças e } \\
\text { agravos no contexto da APS, incluindo abordagem } \\
\text { racional de sintomas, eficácia de tratamentos, } \\
\text { tomada de decisão clínica e qualidade do } \\
\text { atendimento; } \\
\text { - Pesquisas sobre condições raras hereditárias/ } \\
\text { genéticas na atenção primária; } \\
\text { - Educação médica e educação permanente voltada } \\
\text { para a solução de problemas de saúde }\end{array}$ & $\begin{array}{l}\text { - Estudos epidemiológicos } \\
\text { longitudinais em medicina de } \\
\text { família. } \\
\text { - Estudos pragmáticos de } \\
\text { intervenções clínicas na APS. } \\
\text { - Levantamento e análise de } \\
\text { abordagens na atenção primária. } \\
\text { - Pesquisas com métodos mistos. }\end{array}$ \\
\hline $\begin{array}{l}\text { Orientação } \\
\text { comunitária }\end{array}$ & $\begin{array}{l}\text { - Análise do efeito de diferentes modelos e } \\
\text { intervenções em atenção primária na resposta às } \\
\text { necessidades individuais de saúde no contexto local } \\
\text { e das necessidades de saúde das comunidades, além } \\
\text { de possíveis conflitos entre estas duas. }\end{array}$ & $\begin{array}{l}\text { - Pesquisas survey } \\
\text { - Estudos de coorte } \\
\text { observacionais. } \\
\text { - Pesquisas com métodos mistos. }\end{array}$ \\
\hline
\end{tabular}

Fonte: Adaptado da série de artigos Research agenda for general practice/family medicine and primary health care in Europe.

programas de MFC devam estar vinculados à área Medicina I da CAPES. Embora, como já destacamos, ainda não exista uma subárea de MFC dentro desta área, este não é um fator impeditivo para o surgimento de cursos de pós-graduação stricto sensu voltados para esta disciplina. Como apontado pelo mais recente documento da área
Medicina ${ }^{36}$, a maioria de seus 175 cursos de pósgraduação de mestrado acadêmico e profissional e doutorado acadêmico adotam denominações abrangentes como Ciências Médicas e Ciências da Saúde. Mesmo quando designados por uma disciplina clínica específica como cardiologia ou endocrinologia, suas dissertações, teses e publi- 
cações têm sido "de caráter interdisciplinar, de interesse a subáreas diversas da especialidade mé-

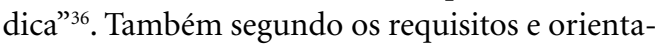
ções desta área ${ }^{37}$, a estrutura curricular de novos programas "deve ser prioritariamente de caráter metodológico" e "serão valorizadas iniciativas de caráter multidisciplinar" ${ }^{\text {"37. }}$.

Assumindo essa ênfase metodológica e interdisciplinar, é preciso destacar que o escopo clínico da MFC é amplo, porém, tem como traço singular a definição de uma população de referência e a interação entre os aspectos psicossociais e biomédicos, no nível individual, familiar e comunitário tanto na produção do adoecimento quanto dos dispositivos de cuidado ${ }^{38}$. Ian McWhinney ${ }^{29}$ já apontava em 1966 que a pesquisa em medicina de família deveria ter como principais focos: a epidemiologia das doenças na atenção primária, o estudo de aspectos clínicos dos problemas de saúde - como avaliação de sintomas, sinais e testes diagnósticos - e seus aspectos psicossociais.

Assim, programas de mestrado em MFC devem possibilitar ao estudante conhecer as principais abordagens metodológicas disponíveis para a pesquisa clínica (epidemiológica, diagnósticoterapêutica e qualitativa) e, em seguida, subsidiá-lo para identificar quais destas melhor se aplica à sua questão de estudo, oferecendo também módulos e disciplinas optativas de aprofundamento considerando o método escolhido. Deve haver espaço no currículo para a aprendizagem em métodos mistos de investigação, considerando que a população de estudo de um ou uma MFC, especialmente aquele ou aquela que pretenda integrar sua pesquisa à sua prática assistencial, é potencialmente pequena, porém, rica em quantidades de informações e variáveis vinculadas aos seus contextos clínico e social e que demandam tanto recortes quantitativos quanto qualitativos de análise. A análise dos impactos dos determinantes sociais em saúde no cenário clínico da APS e de aspectos relacionados à epidemiologia e serviços de saúde - particularmente o conceito From Evidence to Decision Making ${ }^{39}$ - também deve ser incorporada aos currículos dos programas.

As referências que adotamos para a elaboração destas sugestões também apontam para a importância de dois núcleos de competências/ conteúdos que devem ser abordados em disciplinas de programas de mestrado em MFC: comunicação clínica/cuidados centrados no paciente e ensino-aprendizagem em MFC. Como destacado pelo currículo da Western University, um componente essencial da clínica em MFC é a abordagem dos aspectos psicossociais envolvidos nos proces- sos de saúde, adoecimento e cuidado, que estão presentes no método clínico centrado na pessoa. As competências para essa abordagem devem ser desenvolvidas através de estudo teórico e treinamento de habilidades em técnicas e ferramentas de comunicação clínica centradas no paciente, tanto na graduação médica quanto nos programas de residência em MFC. O constante aprimoramento dessas competências, sua aprendizagem e avaliação em desfechos clínicos e de centralidade no paciente são questões fundamentais de pesquisa para o desenvolvimento da MFC como disciplina acadêmica e como recurso de cuidado em saúde. Na mesma direção, o aumento do espaço da formação em APS no currículo da graduação médica, o crescimento significativo da oferta de vagas de residência em MFC e o surgimento de outras estratégias de especialização em MFC e APS que têm sido propostas para suprir o déficit desses profissionais no Brasil tornam também relevantes e necessárias a descrição e a avaliação dessas experiências de ensino-aprendizagem em curso no âmbito nacional ${ }^{1,7,13}$. Essas pesquisas devem ser impulsionadas pela presença regular deste tema em disciplinas de programas de mestrado em MFC no Brasil.

Neste sentido, apontamos para a necessidade de um currículo de mestrado em MFC em 2 anos (como exigido pela CAPES) formado principalmente por disciplinas de introdução à teoria e pesquisa em medicina de família, pesquisa em cuidados centrados no paciente com ênfase em comunicação clínica, pesquisa em ensino-aprendizagem em MFC, além de uma ou mais disciplinas de métodos de pesquisa, que devem estar concentradas no primeiro ano do curso. Disciplinas optativas de tópicos especiais podem ser oferecidas no segundo semestre ou, eventualmente, terceiro semestre, e abordar recortes específicos de acordo com as linhas de pesquisa de cada programa e expertise de seus orientadores. A proposta de currículo está apresentada no Quadro 3. Dada a possibilidade de cursos de mestrado profissional no Brasil, sugerimos que os primeiros programas se enquadrem nesta modalidade, permitindo que os médicos e médicas de família possam conciliar sua prática assistencial e docente, com a formação em pesquisa. Além disso, a modalidade profissional demanda dos cursos de pós-graduação o desenvolvimento de produtos finais que contribuam para o manejo de problemas e desafios de instituições e atores sociais em sua área de conhecimento, potencializando, no caso da MFC, o impacto de cursos de mestrado em MFC na qualificação da atenção primária à 
Quadro 3. Proposta de organização curricular de um curso nacional de mestrado em medicina de família e comunidade.

\begin{tabular}{|l|l|}
\hline \multicolumn{1}{|c|}{ Disciplinas obrigatórias } & \multicolumn{1}{c|}{ Princípios de ementa } \\
\hline $\begin{array}{l}\text { Fundamentos teóricos da Medicina de } \\
\text { Família e Comunidade }\end{array}$ & $\begin{array}{l}\text { Epistemologia das ciências da saúde. Epistemologia de campos } \\
\text { interdisciplinares, teoria dos sistemas e complexidade. História da } \\
\text { MFC moderna. MFC como campo de conhecimento e disciplina } \\
\text { acadêmica. }\end{array}$ \\
\hline $\begin{array}{l}\text { Introdução à pesquisa em Medicina } \\
\text { de Família e Comunidade: recortes } \\
\text { epistêmicos e métodos de investigação. }\end{array}$ & $\begin{array}{l}\text { Temas e métodos de pesquisa em MFC. Análise de pesquisas e } \\
\text { publicações de alto impacto em MFC. Elaboração de um projeto } \\
\text { de pesquisa em MFC. Ética em pesquisa. }\end{array}$ \\
\hline $\begin{array}{l}\text { Pesquisa em comunicação clínica e } \\
\text { cuidados centrados no paciente }\end{array}$ & $\begin{array}{l}\text { Cuidados centrados no paciente: princípios e propostas de } \\
\text { operacionalização. Comunicação clínica: teorias e técnicas. } \\
\text { Métodos e instrumentos de pesquisa para avaliação da } \\
\text { centralidade do paciente. }\end{array}$ \\
\hline $\begin{array}{l}\text { Pesquisa em ensino-aprendizagem em } \\
\text { medicina de família e comunidade }\end{array}$ & $\begin{array}{l}\text { Educação no ensino superior no Brasil. Métodos de ensino } \\
\text { e avaliação na formação médica. Ensino da MFC no Brasil. } \\
\text { Avaliação de métodos e experiências de ensino-aprendizagem. }\end{array}$ \\
\hline $\begin{array}{l}\text { Metodologias de pesquisa em medicina } \\
\text { de família e comunidade (organização em } \\
\text { uma ou mais disciplinas). }\end{array}$ & $\begin{array}{l}\text { Pesquisa epidemiológica em cenários de atenção primária à } \\
\text { saúde; pesquisa sobre intervençães diagnósticas e terapêuticas } \\
\text { em medicina de família; pesquisa qualitativa em saúde; pesquisas } \\
\text { com métodos mistos; avaliação de políticas e serviços em atenção } \\
\text { primária à saúde. }\end{array}$ \\
\hline $\begin{array}{l}\text { Disciplinas optativas } \\
\text { Princípios de ementa } \\
\text { medicina de família e comunidade }\end{array}$ & $\begin{array}{l}\text { Estado da arte em pesquisa de acordo com recortes temáticos das } \\
\text { linhas de pesquisa e trabalhos de orientadores de cada programa. }\end{array}$ \\
\hline
\end{tabular}

Fonte: Os autores.

saúde ofertada à população brasileira e na formação de profissionais competentes e socialmente engajados nesse campo.

\section{Os desafios para a construção de um $s$ istema de pós-graduação stricto sensu em atenção primária à saúde e medicina de família e comunidade no Brasil}

Como buscou-se mostrar nas sessões anteriores, a constituição de um sistema de pós-graduação stricto sensu em determinada área não é tarefa simples. Apesar de não ser estritamente necessária a institucionalização de uma área de conhecimento junto à CAPES para que programas sejam criados, tal movimento contribui para a visibilidade social da área, o que pode se associar a maiores investimentos. Neste texto, sugerimos a área Medicina I como aquela que provavelmente melhor se ajuste a todas as necessidades e particularidades da MFC. Contudo, a avaliação dos programas de pós-graduação nas áreas médicas possuem critérios que, dada a formação majoritária dos MFC em nível de doutorado ser na área de saúde coletiva, podem dificultar a composição de um bom corpo docente de MFC.
O cenário universitário é essencial para a criação de programas de mestrado em MFC, dada sua vocação por definição na produção de conhecimento. Inserir com potência a MFC na universidade é um processo gradual de sensibilização para a importância da especialidade para as práticas em saúde e tensionamento de resilientes relações de poder historicamente constituídas no âmbito da medicina e da universidade. Atrelado a isso, a insuficiência de docentes MFC nestas instituições, em particular nas públicas, agravada pela ainda pequena proporção de MFC com doutorado e falta de estruturas departamentais que deem ancoragem a estas iniciativas, exigem investimentos de médio e longo prazo. Contudo, nenhum programa de pós-graduação sobrevive apenas com profissionais de suas respectivas áreas.

A integração de docentes de diversas áreas é fundamental. Estatísticos, cientistas sociais, engenheiros biomédicos e profissionais de saúde de distintas categorias com interesse e linhas de pesquisa em APS devem contribuir na formação de mestres, trazendo conhecimentos metodológicos e interdisciplinares relevantes para as pesquisas a serem desenvolvidas na pós-graduação. 
Por outro lado, poucas universidades no país têm número de MFC razoável para a criação de um programa de mestrado em MFC com corpo docente robusto da especialidade. Na maior parte delas, movimentos uni-institucionais resultarão em programas com muito poucos MFC em seu quadro docente permanente. Tal realidade tende a reforçar o quadro descrito de concentração da pós-graduação nos grandes centros urbanos das regiões sudeste e sul do país. O movimento de incorporar docentes de áreas distintas é salutar nesse sentido, mas deve ser feito no sentido de não obscurecer a MFC. É essencial que o mestre em MFC formule questões de pesquisa e empreenda práticas pedagógicas que explorem aspectos fundamentais da especialidade e da APS. Portanto, os múltiplos saberes - métodos qualitativos de pesquisa, bioestatística, epidemiologia, ética, etc. - precisam entrar em cena a partir de uma matriz teórica e um campo de possibilidades de reflexão bem constituído a partir da realidade da MFC. Para além deste movimento interdisciplinar de constituição do corpo docente do programa, também se deve investir em redes de pesquisa interinstitucionais, incorporação de docentes visitantes e colaboradores e internacionalização.

\section{Considerações finais}

Nosso objetivo, com este trabalho, foi reunir informações e sugestões que possam subsidiar e impulsionar o surgimento de programas de mestrado e, futuramente, de programas de doutorado em medicina de família e comunidade no Brasil. A educação superior no Brasil atravessa um momento complexo de debates e propostas que passam por medidas que apontam para a redução da participação do Estado, especialmente na pós-graduação stricto sensu $u^{40}$, em que as universidades públicas brasileiras têm exercido historicamente um papel protagonista e de valor reconhecido internacionalmente. No caso das ciências da saúde, consideramos prejudicial e contrária aos princípios constitucionais qualquer tendência de diminuição da liderança e da presença do Estado e do ensino público na formação profissional em saúde, seja na graduação ou na pós-graduação. A saúde é um direito social garantido na constituição brasileira, mediado pelo maior sistema público de saúde, o SUS, que possui como uma de suas áreas de atuação a ordenação da formação de recursos humanos na área de saúde. $\mathrm{Na}$ atenção primária à saúde, cujo principal pilar no Brasil é a Estratégia Saúde da Família, uma ampla e majoritária participação do Estado no financiamento e execução de suas ações assistenciais e formativas é indispensável. Portanto, é fundamental que o desenvolvimento de programas de pós-graduação stricto sensu em medicina de família e comunidade no país seja tomado como uma agenda prioritária, tanto dos setores de saúde quanto da educação, pelo Estado brasileiro, através de linhas de financiamento e editais específicos.

As sugestões curriculares aqui apresentadas pretendem apenas ser um ponto de partida para análises e desenvolvimentos posteriores de instituições envolvidas no ensino e aprendizagem em medicina de família no Brasil. Destacamos entre estas: o grupo de trabalho de ensino-aprendizagem da Sociedade Brasileira de Medicina de Família e Comunidade (SBMFC), departamentos de medicina de família e comunidade e docentes médicos e médicas de família e comunidade das instituições de ensino superior do Brasil, além de instituições e atores da educação médica em geral, de outras especialidades e áreas de conhecimento afins à medicina de família e comunidade, além dos próprios Ministérios da Saúde e da Educação do Brasil. Certamente, toda sugestão curricular de pós-graduação stricto sensu, voltada a contribuir para um universo tão rico e heterogêneo como a atenção primária brasileira, deve ser flexível para adaptações às necessidades e singularidades de contextos locais. Além disso, nossa exploração inicial do cenário internacional da pós-graduação stricto sensu em MFC também sinaliza a necessidade do levantamento e disponibilização de informações e orientações mais precisas, tanto para dar suporte a novos programas quanto para a avaliação e aprimoramento dos já existentes. Esses subsídios devem ser reunidos e elaborados no âmbito regional pela Confederação Ibero-Americana de Medicina Familiar (CIMF) e mundial, pela WONCA e pela WFME.

Cabe também refletir para a necessária expansão qualitativa e quantitativa da produção acadêmica dos MFC, em particular daquela com foco na especialidade e na APS, o que não é simples diante de um cenário social de fragilização de políticas públicas, precarização do sistema de saúde público, cortes orçamentários e desvalorização da universidade. Aqui também torna-se essencial a constituição de redes de pesquisa, tanto entre serviços de saúde, quanto entre instituições de ensino superior. À MFC apresenta-se a árdua tarefa de ao mesmo tempo ter dois complexos cenários a serem fortalecidos: o serviço de saúde em APS, seja público ou privado; e a academia, 
seja em nível de graduação ou pós-graduação. O Brasil apresenta um cenário educacional e de saúde propício e demandante do surgimento de programas de mestrado em medicina de família e comunidade. Propostas e iniciativas como as aqui elencadas são necessárias para sua efetivação.

\section{Colaboradores}

LD Wenceslau e TD Sarti colaboraram igualmente na concepção e realização do estudo, e na redação da versão inicial do manuscrito. TG Trindade realizou revisão crítica da primeira versão e contribuições para a versão final do trabalho.

\section{Referências}

1. Mendonça MHM, Matta GC, Gondim R, Giovanella L, organizadores. Atenção Primária à Saúde no Brasil: conceitos, práticas e pesquisa. Rio de Janeiro: Editora Fiocruz; 2018

2. Brasil. Agência Nacional de Saúde Suplementar (ANS). Laboratórios de inovação sobre experiências em atenção primária na saúde suplementar. Rio de Janeiro: ANS; 2018.

3. Brasil. Ministério da Saúde (MS). e-Gestor Atenção Básica. Histórico de cobertura. [página na internet]. 2019. [acessado 2019 Out 08]. Disponível em: https:// egestorab.saude.gov.br/paginas/acessoPublico/relatorios/relHistoricoCobertura.xhtml

4. Bastos ML, Menzies D, Hone T, Dehghani K, Trajman A. The impact of the Brazilian family health strategy on selected primary care sensitive conditions: a systematic review. PLoS One 2017; 12(8):e0182336.

5. Rasella D, Harhay MO, Pamponet ML, Aquino R, Barreto ML. Impact of primary health care on mortality from heart and cerebrovascular diseases in Brazil: a nationwide analysis of longitudinal data. BMJ 2014; 3(349):g4014.

6. Hone T, Rasella D, Barreto ML, Majeed A, Millett C. Association between expansion of primary healthcare and racial inequalities in mortality amenable to primary care in Brazil: a national longitudinal analysis. PLoS Med 2017; 14(5):e1002306.

7. Augusto DK, David L, Oliveira DOPS, Trindade TG, Lermen Junior N, Poli Neto P. Quantos médicos de família e comunidade temos no Brasil? Rev Bras Med Fam Comunidade 2018; 13(40):1-4.

8. White KL. Fundamental research at primary care level. Lancet 2000; 355(9218):1904-1906.

9. Fontenelle LF, Rossi SV, Oliveira MHM, Brandão DJ, Sarti TD. Postgraduate education among family and community physicians in Brazil: the Trajetórias MFC project. medRxiv 2019; 19005744.

10. Sparks BL, Gupta SK. Research in family medicine in developing countries. Ann Fam Med 2004; 2(Supl. 2):S55-S59.

11. Goodyear-Smith F, Mash B. What makes research primary care research? In: Goodyear-Smith F, Mash B, editors. How to Do Primary Care Research. New York: CRC Press; 2019. p. 3-6.
12. Falk J. A Medicina de Família e Comunidade e sua entidade nacional: histórico e perspectivas. Rev Bras Med Fam Comunidade 2004; 1(1):5-10.

13. Scheffer M, Cassenote A, Guilloux AGA, Biancarelli A, Miotto BA, Mainardi GM. Demografia Médica no Brasil 2018. São Paulo: Conselho Federal de Medicina; 2018.

14. Almeida KNC. A pós-graduação no Brasil: história de uma tradição inventada [tese]. São Paulo: Universidade Estadual de Campinas; 2017.

15. Balbachevsky E. A pós-graduação no Brasil: novos desafios para uma política bem-sucedida. In: Brock C, Schwartzman S, organizadores. Os desafios da educação no Brasil. Rio de Janeiro: Nova Fronteira; 2005. p. 285-314.

16. Cirani CBS, Campanario MA, Silva HHM. A evolução do ensino da pós-graduação senso estrito no Brasil: análise exploratória e proposições para pesquisa. Avaliação (Campinas) 2015; 20(1):163-187.

17. Brasil. Coordenação de Aperfeiçoamento de Pessoal de Nível Superior (Capes). GeoCapes: Sistema de Informações Georreferenciadas [página na internet]. 2019. [acessado 2019 Out 08]. Disponível em: https:// geocapes.capes.gov.br/geocapes/

18. Gatti BA. Reflexão sobre os desafios da pós-graduação: novas perspectivas sociais, conhecimento e poder. Rev. Bras. Educ. 2001; (18):108-116.

19. Andrade RO. Desafios para além da pós-graduação: recém-doutores enfrentam dificuldades para ingressar no mercado de trabalho. Pesquisa Fapesp 2018; 271.

20. Camargo Jr KR. Produção científica: avaliação da qualidade ou ficção contábil? Cad Saude Publica 2013; 29(9):1707-1711.

21. Coordenação de Aperfeiçoamento de Pessoal de Nível Superior (CAPES). Relatório de Avaliação 2013-2016 quadrienal 2017. Área de avaliação: Saúde Coletiva. Brasília: CAPES; 2017.

22. Contribuição para uma agenda política estratégica para a Atenção Primária à Saúde no SUS. Saúde Debate 2018; 42(spe. 1):406-430. 
23. Fassa A, Teixeira CP, Fassa E, Pinto ME, Costa M, Antero MF. Projeto Político Pedagógico do Mestrado Profissional em Saúde da Família - Profsaúde. Rio de Janeiro: Abrasco; 2016.

24. Organisation for Economic Cooperation and Development (OECD). Health at a Glance 2017: OECD Indicators. Paris: OECD Publishing; 2017.

25. Pérez PB, López-Valcárcel BG. Estimación de la oferta y demanda de Médicos Especialistas. España 2018-2030. Equipo Economía de la Salud. Universidad de Las Palmas de Gran Canaria. [acessado 2019 Out 08]. Disponível em: https://www.mscbs.gob.es/profesionales/ formacion/necesidadEspecialistas/doc/20182030

26. Couper I, Mash B, Smith S, Schweitzer B. Outcomes for family medicine postgraduate training in South Africa. South African Family Practice 2012; 54(6):501506.

27. Jenn NC, Lieng Teng C, Abdullah A, Hoong Wong C, Sherina Hanafi N, Su Yin Phoa S, Tong WT. The status of Family Medicine training programs in the Asia Pacific. Fam Med 2016; 48(3):194-202.

28. World Organization of National Colleges, Academies and Academic Associations of General Practitioners / Family Physicians (WONCA). WONCA Global Standards for Postgraduate Family Medicine Education. A guide for program development. 2016. [acessado 2019 Out 08]. Disponível em: http://www.globalfamilydoctor.com/site/DefaultSite/filesystem/documents/ Groups/Education/WONCA\%20Global\%20Standards $\% 20$ for $\% 20$ Postgraduate $\% 20$ Family $\% 20$ Medicine\%20Guide\%20for\%20Program\%20Development\%202016.pdf

29. Hummers-Pradier E, Beyer M, Chevallier P, Eilat-Tsanani S, Lionis C, Peremans L, Petek D, Rurik I, Soler JK, Stoffers HE, Topsever P, Ungan M, Van Royen $\mathrm{P}$. The research agenda for general practice/family medicine and primary health care in in Europe. Part 1. Background and methodology. Eur J Gen Pract 2009; 15(4):243-250.

30. Hummers-Pradier E, Beyer M, Chevallier P, Eilat-Tsanani S, Lionis C, Peremans L, Petek D, Rurik I, Soler JK, Stoffers HE, Topsever P, Ungan M, van Royen $\mathrm{P}$. The research agenda for general practice/family medicine and primary health care in Europe. Part 2. Results: Primary care management and community orientation. Eur J Gen Pract 2010; 16(1):42-50.

31. Van Royen P, Beyer M, Chevallier P, Eilat-Tsanani S, Lionis C, Peremans L, Petek D, Rurik I, Soler JK, Stoffers HE, Topsever P, Ungan M, Hummers-Pradier E. The research agenda for general practice/family medicine and primary health care in Europe. Part 3. Results: Person centred care, comprehensive and holistic approach. Eur J Gen Pract 2010; 16(2):113-119.
32. Hummers-Pradier E, Beyer M, Chevallier P, Eilat-Tsanani S, Lionis C, Peremans L, Petek D, Rurik I, Soler JK, Stoffers HE, Topsever P, Ungan M, van Royen $\mathrm{P}$. The research agenda for general practice/family medicine and primary health care in Europe. Part 4. Results: Specific problem solving skills. Eur J Gen Pract 2010; 16(3):174-181.

33. Van Royen P, Beyer M, Chevallier P, Eilat-Tsanani S, Lionis C, Peremans L, Petek D, Rurik I, Soler JK, Stoffers HE, Topsever P, Ungan M, Hummers-Pradier E. The research agenda for general practice/family medicine and primary health care in Europe. Part 5: Needs and implications for future research and policy. Eur J Gen Pract 2010; 16(4):244-248.

34. Brasil. LDB: Lei de diretrizes e bases da educação nacional. Brasília: Senado Federal; 2017.

35. Brasil. Resolução no 7, de 11 de dezembro de 2017. Estabelece normas para o funcionamento de cursos de pós-graduação stricto sensu. Diário Oficial da União 2017; 12 dez.

36. Coordenação de Aperfeiçoamento de Pessoal de Nível Superior (CAPES). Documento de Área. Área 15: Medicina I. Brasília: CAPES; 2019.

37. Coordenação de Aperfeiçoamento de Pessoal de Nível Superior (CAPES). Requisitos e orientações para propostas de cursos novos: mestrado e doutorado acadêmicos e profissionais. Área 15: Medicina I. Brasília: CAPES; 2016.

38. Fetters MD, Guetterman TC. Discovering and doing family medicine and community health research. Fam Med Com Health 2019; 7:e000084.

39. Poot C, van der Kleij R, Brakema E, Vermond D, Williams S, Cragg L, van den Broek JM, Chavannes NH. From research to evidence-informed decision making: a systematic approach. J Public Health (Oxford) 2018; 40(Supl. 1):i3-i12.

40. Angelo C. Brazil's government freezes nearly half of its science spending. Nature 2019; 568(7751):155-156.

Artigo apresentado em 10/10/2019

Aprovado em 23/10/2019

Versão final apresentada em 25/10/2019 